\title{
Soft Computing for Vehicle Side Slip Angle Based on Kalman Filter
}

\author{
Sun Qiuyun \\ School Management and Information \\ Shandong Transport Vocational College \\ Weifang, P.R.China \\ shenknight@yahoo.com.cn
}

\begin{abstract}
To solve the problem that vehicle side slip angle in vehicle control process is too difficult to measure on-line, soft computing for vehicle side slip angle is established with Kalman filter and driver-vehicle closed-loop system, based on parameter soft sensor theory and discrete signal filtering theory. This measurement can realize one mapping from information set of yaw rate and lateral acceleration which is easy to measure to side slip angle. The contrastive results of simulation and road test verify the effectiveness and higher precision of this measurement. And the application of soft computing technology has provided a feasible and accuracy and effective way for the estimator design in vehicle control system. The realization of state estimation based on soft computing technology provides a theoretical basis on soft computing applied to vehicle stability control system.
\end{abstract}

Index Terms - vehicle side slip angle, soft computing, Kalman filter, state estimation

\section{INTRODUCTION}

The vehicle road ability is always the focus. To this end, the researchers have developed a series of vehicle electronic control systems, such as anti-lock braking system, acceleration slip regulation, traction control system, etc. These electronic control systems effectively improve vehicle stability performance by controlling the key state parameters of the moving vehicle ${ }^{[1,2]}$. The premise and necessary condition to improve stability performance is measured accurately of vehicle state parameters which can reflect the vehicle itself changes in the control process. Yaw rate and vehicle side slip angle are important parameters to vehicle control system ${ }^{[3,4]}$, the former can be directly measured through the corresponding sensors, and the latter is difficult or expensive to directly measure. Usually vehicle side slip angle was estimated by other vehicle state parameters (such as vehicle longitudinal velocity and lateral velocity, and so on). One is to direct integral method to calculate vehicle side slip angle, but the noise signal is also involved in the integration process, easy to cause greater error $^{[5,6]}$. Another is to use GPS and gyroscope to measure, but the cost is too high to promote ${ }^{[7,8]}$. In addition, some soft computing based on state estimation has good application in the field ${ }^{[9-11]}$. To solve the problem that vehicle side slip angle in vehicle control process is too difficult to measure on-line, the vehicle side slip angle's soft computing model is established with Kalman filter and driver-vehicle closed-loop system, based on parameter soft sensor theory and discrete signal filtering theory. This measurement can realize the on- line inference and estimation of side slip angle by using of yaw rate and lateral acceleration which is easy to measure. Thus, the soft computing of side slip angle is achieved through state estimation. In this paper, based on the preview optimal control theory of curvature and the preview follow theory, the soft computing model was build by a two degree of freedom driverautomobile closed-loop system model. Its state equation and observation equation have been derived and discrete to draw the system discrete observation equation on the observation quantity of yaw rate and lateral acceleration, and to do the simulation.

\section{SOFT COMPUTING}

To effectively solve the information incompleteness in the control process, the soft computing has been widely applied as a kind of effective method. In 1978, Brosillow C.B. put forward a new inference control of the unpredictable variables, and gradually formed the soft sensor theory and its application technology in the field of process control ${ }^{[12]}$. Especially professor McaVoy who is a famous expert in the international process control field is considered that soft computing is one of broad directions need to study in the future control field, makes the soft computing further research work ${ }^{[13]}$.

The based idea of soft computing is the application of computer technology, to the important variables (or called dominant variable) which are difficult to measure or immeasurable, select some other easily measured variables (or called instrumental variables), and through the establishment of mathematical model of instrumental variables which is input and dominant variable which is output by on-line state estimation. Thus, software replaces hardware.

Its core is to establish a soft computing model which can convey the mathematical relationship between instrumental variables and dominant variable ${ }^{[12]}$. To complete the mapping from easy measurement information set which consists of instrumental variables to dominate variable, as shown in figure 1 .

There are different kinds of modeling methods and cross with each other, including mechanism modeling, state estimation, regression analysis, pattern recognition, artificial neural network and fuzzy mathematics and genetic algorithm, etc. In this paper, adapt state estimation method, establish vehicle soft computing model and make use of Kalman filter 
algorithm to derive the estimated value of dominant variable from the instrumental variables.

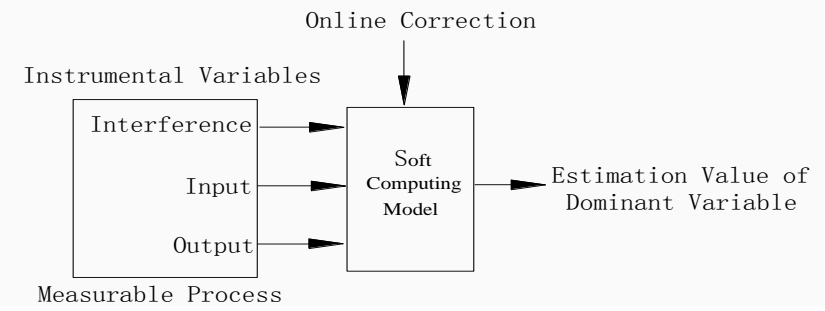

Fig. 1. The chart of soft computing model

The key question of state estimation based on Kalman filtering is transformed parameter measurement into state observation and state estimation, that is, get immeasurable variables from observable variables by the arithmetic. Kalman filter was developed by Rudolph Kalman since $1960^{[14]}$; it represents one system state space structure and state change over time with filtering random disturbance. Kalman filter has works well and theoretically attractive. It is shown that Kalman filter is the effective way to obtain the minimum variance of the estimation error. In this paper, the soft computing of vehicle side slip angle based on Kalman filtering is transformed the computing into a state estimation, taking the information of yaw rate and lateral acceleration as instrumental variables, and side slip angle to be estimate as dominant variable.

\section{MODEL FOUNDATION}

\section{A. State Estimation Model}

Based on the preview optimal control theory of curvature and the preview follow theory ${ }^{[15]}$, the soft sensor model was build by a two degree of freedom driver-automobile closedloop system model. as shown in figure 2 .

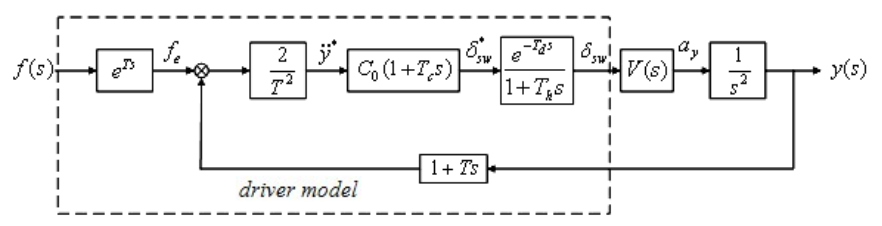

Fig. 2. Driver-automobile closed-loop system model

State equation:

$\left[\begin{array}{c}\dot{r} \\ \dot{\beta} \\ \dot{y} \\ \dot{v}_{a} \\ \dot{x}_{1} \\ \dot{x}_{2}\end{array}\right]=\left[\begin{array}{cccccc}a_{11} & a_{12} & 0 & 0 & a_{15} & a_{16} \\ a_{21} & a_{22} & 0 & 0 & a_{25} & a_{26} \\ 0 & 0 & 0 & 1 & 0 & 0 \\ a_{41} & a_{42} & 0 & 0 & a_{45} & a_{46} \\ 0 & 0 & 0 & 0 & 0 & 1 \\ a_{61} & a_{62} & a_{63} & a_{64} & a_{65} & a_{66}\end{array}\right]\left[\begin{array}{c}r \\ \beta \\ y \\ v_{a} \\ x_{1} \\ x_{2}\end{array}\right]+\left[\begin{array}{c}0 \\ 0 \\ 0 \\ 0 \\ 0 \\ b_{6}\end{array}\right] f_{r}$

Output equation: $\left[\begin{array}{c}y \\ a_{y} \\ \delta_{s w} \\ \dot{\delta}_{s w} \\ S_{1} \\ S_{2}\end{array}\right]=\left[\begin{array}{cccccc}0 & 0 & c_{13} & 0 & 0 & 0 \\ c_{21} & c_{22} & 0 & 0 & c_{25} & c_{26} \\ 0 & 0 & 0 & 0 & c_{35} & c_{36} \\ c_{41} & c_{42} & c_{43} & c_{44} & c_{45} & c_{46} \\ c_{51} & c_{52} & 0 & 0 & c_{55} & c_{56} \\ c_{61} & c_{62} & 0 & 0 & 0 & 0\end{array}\right]\left[\begin{array}{c}r \\ \beta \\ y \\ v_{a} \\ x_{1} \\ x_{2}\end{array}\right]+\left[\begin{array}{c}0 \\ 0 \\ 0 \\ d_{4} \\ 0 \\ 0\end{array}\right] f_{r}$

In the above equations, $f_{r}=f(t+T)+T_{c} \dot{f}(t+T), r$ is yaw rate, $\beta$ is side slip angle, $v_{a}$ is the value of vehicle absolute center-of-mass velocity in the $Y$ direction of the absolute coordinate system. $S_{1}$ and $S_{2}$ are front and rear wheel lateral force coefficient respectively, $x_{1}$ and $x_{2}$ are two state variables in the model.

To state estimate by Kalman filter computing, the state equation and output equation demand continuous system discretization. The sample cycle is $0.01 \mathrm{~s}$. There is noise $w$ in the discrete state equations and noise $v$ in the discrete output equations, and we assume that noise $w$ and noise $v$ are an independent zero mean random Gaussian white noise sequence. $A_{d} 、 B_{d} 、 C_{d}$ are system matrix, input matrix and measurement matrix after discretization, respectively. Then the discrete state equation for the closed-loop system is written as:

$$
\left[\begin{array}{c}
r(k+1) \\
\beta(k+1) \\
y(k+1) \\
v_{a}(k+1) \\
x_{1}(k+1) \\
x_{2}(k+1)
\end{array}\right]=A_{d}\left[\begin{array}{c}
r(k) \\
\beta(k) \\
y(k) \\
v_{a}(k) \\
x_{1}(k) \\
x_{2}(k)
\end{array}\right]+B_{d} f_{r}(k)+w(k)
$$

The discrete system observation equation in which yaw rate and lateral acceleration are the observed quantity is written as:

$$
\left[\begin{array}{c}
r(k) \\
a_{y}(k)
\end{array}\right]=C_{d}\left[\begin{array}{c}
r(k) \\
\beta(k) \\
y(k) \\
v_{a}(k) \\
x_{1}(k) \\
x_{2}(k)
\end{array}\right]+v(k)
$$

\section{B. Kalman Filter}

Kalman filter is an algorithm that uses a series of measurements observed over time, containing random noise and other inaccurate factors, and estimates the unknown variables that tend to be more precise than those based on a single measurement alone. More formally, Kalman filter operates recursively on streams of noisy input data to produce a statistically optimal estimate of the underlying system state. 
The algorithm works in a two-step process ${ }^{[14]}$. One is predict, another is update. During the computing, Kalman filter produces estimates of the current state variables, along with their uncertainties. Once the outcome of the next measurement (including random noise) is observed, these estimates are updated using a weighted average, with more weight being given to estimates with higher certainty. Because of the algorithm's recursive nature, it can run in real time using only the present input measurements and the previously calculated state; no additional past information is required. The filtering algorithm adopted by this paper is as follows:

- Predict

Predicted state estimate:

$$
x(k+1 \mid k)=A_{d} x(k+1 \mid k)+B_{d} w(k)
$$

Predicted estimate covariance:

$$
P(k+1 \mid k)=A_{d} P(k \mid k) A_{d}^{T}+Q(k)
$$

- Update

Optimal Kalman gain:

$$
K(k+1)=P(k+1 \mid k) C_{d}{ }^{T}\left[C_{d} P(k+1 \mid k) C_{d}{ }^{T}+R(k)\right]^{-1}
$$

Updated state estimate:

$$
\begin{aligned}
x(k+1 \mid k+1)= & x(k+1 \mid k)+ \\
& K(k+1)\left[Z(k+1)-C_{d} x(k+1 \mid k)\right]
\end{aligned}
$$

Updated estimate covariance:

$$
P(k+1 \mid k+1)=\left[I-K(k+1) C_{d}\right] P(k+1 \mid k)
$$

Where, $w(k)$ is the average of system noise, $Q(k)$ is the covariance matrix of system noise, $v(k)$ is the average of observation noise, $R(k)$ is the covariance matrix of observation noise, $Z(k)$ is the measurements of yaw rate and lateral acceleration.

\section{SOFT COMPUTING AND ROAD TEST}

\section{A. Road Test}

Road test has been conducted to gain the data of longitudinal velocity, lateral velocity, yaw rate, lateral acceleration and vehicle side slip angle. The condition of road test is double-shift line (BS ISO 3888-2:2002), the speed of the test vehicle is about $20 \mathrm{~m} / \mathrm{s}$ during the test. The data of yaw rate and lateral acceleration are real-time collected by DMSSGP01 gyroscope, and after successfully recording the data of yaw rate and lateral acceleration, these data that contain noise are the input measurements of state estimation by Kalman filter. Other data are collected by non-contact velocity meter system which consists of LC-761, LC-1100, LC-2100 and LC-5200, and so on, as shown in figure 3. LC-1100 which is a longitudinal velocity sensor and LC-2100 which is a lateral velocity sensor are spatial filter type to collect the data of longitudinal velocity and lateral velocity. The data of side slip angle are measured by using LC-5200 forward distance pulse in the system.

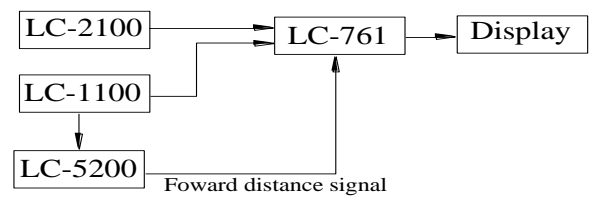

Fig. 3. LC-761 system connection diagram

\section{B. Double-shift Line}

Double-shift line reflects vehicle control performance when avoiding obstacles or overtaking other vehicle s on the road at high speed linear driving. In the process of actual double-shift line driving, the travel route of vehicle should be a smooth curve, as shown in figure 4 . The $\mathrm{X}$-axis represents the direction of vehicle; the Y-axis represents the distance of driver's left side.

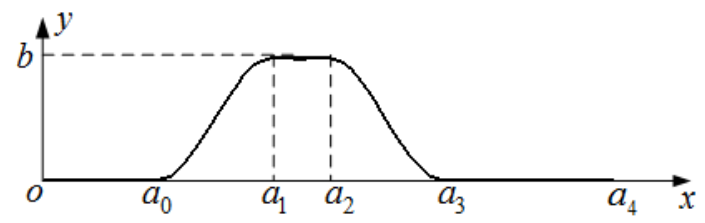

Fig. 4. Fitting of the double-shift line

Next, the test path needs to be fit into a smooth curve by cubic spline function, and get third order curves function which is first derivative continuous. The expression is:

$$
f(t)=\left\{\begin{array}{cc}
0 & \left(0 \leq t<t_{0}\right) \\
g_{0}+g_{1} t+g_{2} t^{2}+g_{3} t^{3} & \left(t_{0} \leq t<t_{1}\right) \\
b & \left(t_{1} \leq t<t_{2}\right) \\
h_{0}+h_{1} t+h_{2} t^{2}+h_{3} t^{3} & \left(t_{2} \leq t<t_{3}\right) \\
0 & \left(t_{3} \leq t \leq t_{4}\right)
\end{array}\right.
$$

Where

$$
\begin{aligned}
& e_{0}=a_{0}^{2}\left(3 a_{1}-a_{0}\right) b /\left(a_{1}-a_{0}\right)^{3} \\
& e_{1}=-6 a_{0} a_{1} b /\left(a_{1}-a_{0}\right)^{3} \\
& e_{2}=3\left(a_{0}+a_{1}\right) b /\left(a_{1}-a_{0}\right)^{3} \\
& e_{3}=-2 b /\left(a_{1}-a_{0}\right)^{3} \\
& g_{0}=e_{0}, \quad g_{i}=e_{i} u^{i}(i=1,2,3) \\
& h_{0}=e_{0}^{\prime}, \quad h_{i}=e_{i}^{\prime} u^{i}(i=1,2,3)
\end{aligned}
$$

\section{Comparison of estimation and road test}

After the text edit has been completed, the paper is ready for the template. Duplicate the template file by using the Save As command, and use the naming convention prescribed by your conference for the name of your paper. In this newly created file, highlight all of the contents and import your prepared text file. You are now ready to style your paper; use the scroll down window on the left of the MS Word Formatting toolbar.

Fig.5 shows the road test data of yaw rate and lateral acceleration that contain noise in the condition of double-shift 
line. These data that contain noise are the input measurements of state estimation by Kalman filter, and then calculated the value of vehicle side slip angle, denoted by $\beta_{k a l}$. The value of vehicle side slip angle has obtained from road test denoted by $\beta_{\text {real }}$. The result of the values' comparison shows that there are the same changing trend and error within $10 \%$ of the test value amplitude, as shown in figure 6 . It is shown that the soft computing can achieve the precise estimation results of vehicle side slip angle and is feasible. The mean error of vehicle side slip angle is $0.0693^{\circ}$ and the standard deviation is $0.0958^{\circ}$.

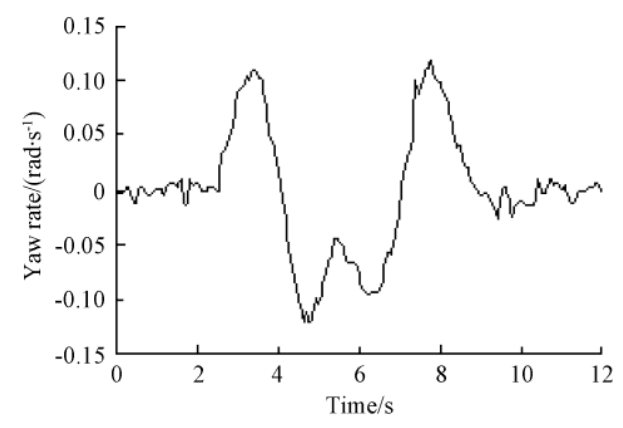

a. Vehicle yaw rate

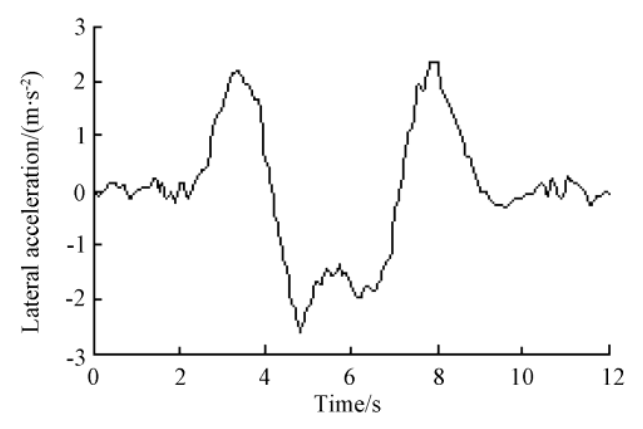

b. Vehicle lateral acceleration

Fig. 5. Test results of double-shift line real vehicle (Condition: BS ISO 3888-2:2002 double-shift line)

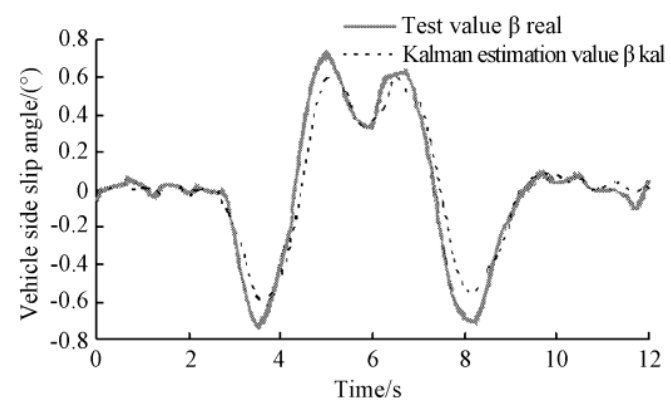

Fig. 6. Comparison of estimation value and test value of side slip angle (Condition: BS ISO 3888-2:2002 double-shift line)

\section{CONCLUSIONS}

To obtain accurate and effective vehicle side slip angle by state estimation, soft computing was introduced in this study. Experimental approach by means of road test was conducted to examine the availability of the computing. Soft computing carried out easily measured parameters to estimate vehicle side slip angle, and the result has higher accuracy. As a result, it is indicated that soft computing can effectively reduce the cost and the state estimation method is reasonable and feasible.

\section{REFERENCES}

[1] Zheng Shuibo, Tang Houjun, Han Zhengzhi,et al. Controller Design for Vehicle Stability Enhancement [J]. Control Engineering Practice, 2006, 14(12):1413- 1421.

[2] Aleksander Hac, Melinda D. Estimation of Vehicle Side Slip Angle and Yaw Rate [J] .SAE Paper. 2000-01-0357. 2000.

[3] Van Zanten A T, Erhardt R, PfaffV G. DC, The vehicle dynamics control system of bosch[C]. SAE 950759, 1995.

[4] Van Zanten A T. Bosch ESP System: 5 Years of Experience [C] .SAE paper 2000-01-1633, 2000.

[5] D Diyabongkarn, R Rajamani, J A Grogg, et al. Development and Experimental Evaluation of a Slip Angle Estimator for Vehicle Stability Control [C]. IEEE 2006. USA: IEEE, Press, 2006.

[6] J Stephant, A Charara, D Meizel. Evaluation of a Sliding Mode Observer for Vehicle Side Slip Angle [J]. Control Engineer Practice, 2007, 15:60-71.

[7] Daily R, Bevly D M. The Use of GPS for Vehicle Stability Control System [J].IEEE Transactions of Industrial Electronics, 2004, 51(2):270-277.

[8] Zhang Xiaolong, Wu Min, Song Jian, et al. Engineering Test Method for Motor Vehicle Yaw Rate and Sideslip Angle Based on GPS Technology [J]. Transactions of the Chinese Society for Agricultural Machinery, 2009, 40(2):27-31. (in Chinese)

[9] Wenzel T A, Burnham K J, Blundell M V. Dual Extended Kalman Filter for Vehicle State and Parameter $[\mathrm{J}]$. Vehicle System Dynamics, 2006, 44( 2) : 153-171.

[10] Wenzel T A, Burnham K J, Blundell M V, et al. Kalman Filter as a Virtual Sensor: Applied to Automotive Stability Systems $[\mathrm{J}]$, Transactions of the Institute of Measurement and Control, 2007, 29 (2): 95-115.

[11] Gustaffson F, Drev M, Forssell U, et al. Virtual Sensors of Tire Pressure and Road Friction [C] . Proceedings of the 2001 Society of Automotive Engineers (SAE) World Congress, March, 2001, Detroit, USA. 2001:010796

[12] C.B. Brosillow. "Inferential control of process", AICHE Journal, 1978, 24, 485-494.

[13] T.J. Mcavoy. Contemplative Stance for Chemical Process Control [J]. Automatica, 1992, 28(2): 441-442.

[14] R. Kalman. A new approach to linear filtering and prediction problems[C]. Trans. ASME-J. Basic. Eng. 82(Series D), 1960:35-45

[15] Lin Fen, Zhao Youqun. Study of a Driver-Automobile Closedloop System's Directional Stability Based on Genetic Algorithm [J]. Mechanical Science and Technology, 2006, 25(10):11511153. (in Chinese) 